\title{
Voice Handicap Index Functional Subscale
}

National Cancer Institute

\section{Source}

National Cancer Institute. Voice Handicap Index Functional Subscale. NCI Thesaurus.

Code C128065.

A subscale of the Voice Handicap Index that measures an individual's perception of the effect of their voice handicap on their daily activity. 